\title{
Best-Response Distributed Subchannel Selection for Minimizing Interference in Femtocell Networks
}

\author{
Shashi Shah* and Somsak Kittipiyakul ${ }^{\dagger}$ \\ ${ }^{*}$ School of Information, Computer and Communication Technology \\ Sirindhorn International Institute of Technology (SIIT), Thammasat University, Pathum Thani 12121, Thailand \\ ${ }^{*}$ School of Information Science, Japan Advanced Institute of Science and Technology (JAIST), Ishikawa 923-1292, Japan \\ Email: *shah.shashi415@gmail.com, ${ }^{\dagger}$ somsak@siit.tu.ac.th
}

\begin{abstract}
We study the problem of channel selection of noncooperative OFDMA femtocells in two-tiered macro-femto networks. Using a physical channel model, we consider a distributed channel allocation of femtocells and a total capacity objective, which is the total capacity of uplink macro users and femto users. We assume a time-slotted system, a time-invariant channel model (no fading), each user knows the signal-to-interferenceplus-noise ratio (SINR) of all channels, and the channel selection happens only at the beginning of each time-slot. We study the performance of a best-response strategy where each user chooses to transmit in the highest-SINR channel. For simplicity, we focus on the homogeneous 3-link, 2-channel case and show that if all users update their actions every time-slot (i.e., all users make simultaneous moves), an oscillation can occur and result in the worst performance. To avoid the oscillation and achieve the highest total capacity, while still assuming no coordination among the users, we propose a stochastic best-response algorithm, where each user updates its channel selection with a selection probability $p$. We use a Markov chain to analyze the average capacity performance and use simulation results to confirm our analysis and also provide performance of other homogeneous cases with more number of homogeneous links and channels. It is shown that the highest total capacity can be achieved when the selection probability $p$ is very small. This stochastic best response with small $p$ in effect provides a sequential move mechanism which requires no coordination among the users and hence avoids the oscillation problem from the simultaneous moves.

Keywords-femtocells; interference management; distributed subchannel selection; simultaneous move; best-response; stochastic best-response, Nash equilibrium.
\end{abstract}

\section{INTRODUCTION}

Femtocells are small, short-ranged (10 to $30 \mathrm{~m})$, low cost, and low powered (10 to $100 \mathrm{~mW}$ ) base stations that aim to provide a better radio coverage to indoor mobile users [1]. It is a plug-and-play device, which can be installed by users at homes/offices similar to $\mathrm{WiFi}$ routers, while it operates in licensed spectrum and provides identical cellular functionality. These indoor access points, referred as femtocell access points (FAPs), create a small wireless coverage area and connect femtocell user equipments (FUEs) to the cellular core network through subscribers broadband internet access. An FAP acts as an access station that enables better voice, data, and multimedia services, mainly in an indoor environment with minimal added equipment costs. These features favor femtocells among mobile operators to achieve an enhanced indoor coverage and capacity. Furthermore, they offload traffic from macro layer of mobile network [2], while achieving an overall reduction in capital and operating expenditures.

The study in [3] estimates that by 2018, approximately 11.5 millions non-residential indoor small cells will be deployed. With such dense deployment, femtocells require high immunity to interference that is prevalent in cellular networks because the spectral resources are limited and shared. Hence, interference management is a critical issue as it affects both system capacity and cellular coverage restrictions [1]. Interferences at both co-tier (between femtocells) and cross-tier (between femtocells and macrocells) possess an increasingly severe threat as deployment of femtocells becomes denser in urban environment, consisting of dense population of network users. The random deployment of femtocells within macrocell coverage, particularly with co-channel spectrum allocation, give rise to severe interference issues. This in turns creates a challenging problem for operators to manage subchannels allocation [4]. The interference in the two-tier macro-femto networks becomes quite different than in conventional cellular networks because of aforementioned issues.

To enable distributed interference management, each femtocell must be aware of its environment and posses selforganizing ability to cope with interferers. As the numbers and location of femtocells become unpredictable, operators should consider decentralized network planning approach allowing the femtocells to self-organize by learning their environment, and gradually change its power and subchannel allocations for interference management.

In this paper we study a best-response game for subchannel selection. We model the problem as a multi-agent noncooperative game, where each user repeatedly best-responds to the others' actions [5]. However, the actions chosen by other users are not indirectly known from the aggregate SINR in each channel. Repeated best-response is a natural, simple, and low-cost behavior to build into distributed systems such as routing and congestion control on the Internet. In many applications, the best-response dynamics eventually drive the system to an equilibrium. However, these results typically rely on the fact that users are synchronized and "take turns" at best-responding to other actions and each user's actions are immediately observable to all other users. Under "traditional" best-response dynamics, agents take turns selecting strategies, each repeatedly selecting a strategy that maximizes his utility 
given the others' current strategies. This goes on until a "stable-state", i.e., a pure Nash equilibrium, is reached [5]. Although a user can keep track of all history of the measured SINRs, we consider a bounded-recall best-response strategy where each user's current action only depends on the current SINRs [6].

Specifically, we propose a distributed OFDMA subchannel selection of non-cooperative femtocells so that the aggregate throughputs of macro and femto users are maximized, i.e., the mutual interferences among the femtocells and macrocells are minimized. We assume that the system is time-slotted and all users are synchronized. Our algorithm does not require that each femto user knows the current strategies of other users, but it only assumes that each femto user knows the SINR of every subchannel at each time-slot.

We model the uplink subchannel allocation in noncooperative femtocells where SINR on candidate subchannels are taken into account. Simulations show different rate of steady-state subchannels allocation that generally depends on femtocell's probability of changing to the candidate subchannel at each transmission time-slot.

Note that the work in this paper can be applied for distributed channel selection, not only to femtocell networks but also to other wireless networks, such as how the secondary users select their channels in cognitive radio networks [7], how a wireless sensor node selects its channel in wireless sensor networks in a factory [8], and how a node selects its channel in body-area-networks [9].

The rest of the paper is organized as follows. Some of the related works are discussed in Section III Section III provides a system model for uplink transmissions in macrofemto networks. Our approach of distributed algorithm for sensing and subchannel allocation is discussed in Section IV Numerical results are provided in Section V. Finally, Section $\mathrm{VI}$ summarizes this paper.

\section{RELATED WORKS}

Recently, many researchers have proposed algorithms for distributed interference management (see, e.g., [10], [11]). In [11], a $Q$-learning approach for self-organizing femtocell networks with no information exchange among users was considered. But provided with situation of dense network users, requirement of maintaining a $Q$-table to make decision have a slower convergence time. Moreover, frequent update of $Q$-table will be required because of randomness in femtocell networks. A decentralized power control scheme for femtocells in a macro-femto environment was proposed in [12] where fairness and interference minimization issues were studied. In [10], subchannels allocation for self-organized femtocells were studied based on best-response strategy that guarantees convergence to a stable Nash equilibrium. However, knowledge about selected subchannels by users was required for subchannel allocations by femtocells.

The channel selection is considered a problem of spectrum sharing. Many algorithms have been proposed based on noncooperative game and Nash equilibrium solutions (see [7],

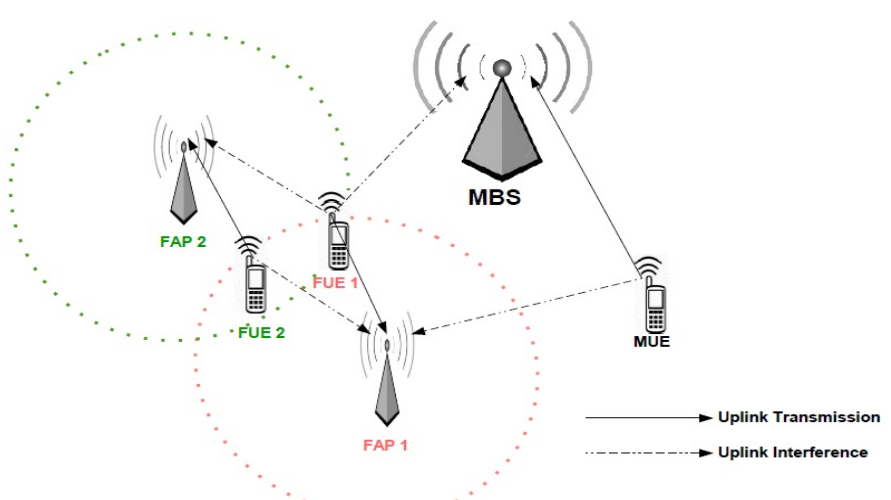

Fig. 1: System model of macro-femto networks. The uplink transmissions and interferences are shown by solid and dashed arrows respectively.

[8], [9]). In [7], the authors studied cognitive radio systems where the secondary users share spectrum with the primary users. In their distributed algorithm, the secondary users are unable to exchange information among each other (i.e., this is a game with incomplete information), and hence rely on the information of charge per unit of bandwidth allocated by primary user.

A related and more general group of works is the distributed power allocation (e.g., [13]). The channel selection problem studied in this paper is a special case of the power allocation. In the channel selection, we assume that the power per subchannel is fixed and constant over subchannels, while the power allocation allows varying power in each subchannel. In [13], the authors proposed an algorithm that assumes exchanges of "interference prices" among the users. Our algorithm does not assume any message exchanges between femtocells or macrocells and hence minimize the communication load.

\section{SySTEM MODEL}

As shown in Figure 1, we assume a co-existence of macro and femto networks in the same area. There are macrocell base stations (MBSs) with corresponding macrocell user equipments (MUEs). In the same area, mostly in indoor area, there are FAPs with corresponding FUEs. For convenience, we focus on the uplink transmissions of the MUEs and FUEs.

We assume a shared frequency spectrum among MUEs and FUEs uplink resources. The shared spectrum bandwidth $B$ of the OFDMA system is divided into $N$ subchannels. For simplicity of representation, we assume one active FUE per FAP. Transmissions of both macrocell and femtocell networks take place on the allocated subchannels of the shared spectrum. During a time-slot, each FUE transmits in a subchannel (similarly for MUE).

Let $M$ and $F$ denote the number of active MUEs and FUEs, respectively. For convenience, we use $M, F$, and $N$ to represent the sets $\{1, \ldots, M\},\{1, \ldots, F\}$, and $\{1, \ldots, N\}$, respectively. 
Let $f \in F$ and $m \in M$ be the uplink transmission links for FUEs and MUEs, respectively. We let $h_{f f, n}$ be the channel gain for transmission on link $f$ (i.e., the channel from FUE $f$ to FAP $f$ over subchannel $n \in N)$. Hence, $h_{f^{\prime} f, n}$ represents the channel gain of the interference from FUE $f^{\prime}$ to FAP $f$ in subchannel $n$. Similar notations are used for MUE and MBS $m$.

In this work, we consider the physical model of the interference. The uplink SINR for link $f$ in subchannel $n$ is given by

$$
\gamma_{f, n}=\frac{p_{f, n} h_{f f, n}}{I_{f, n}+\sigma^{2}}
$$

where $I_{f, n}$ is the uplink co-channel interference of link $f$ in subchannel $n$, i.e., $I_{f, n}$ is the sum of the co-channel interferences from all other FUEs and MUEs using the same subchannel $n$,

$$
I_{f, n}=\sum_{f^{\prime} \neq f} p_{f^{\prime}, n} h_{f^{\prime} f, n}+\sum_{m} p_{m, n} h_{m f, n}
$$

and $\sigma^{2}$ is the additive white Gaussian noise (AWGN) power. Hence, the other subchannels that the femto link $f$ is not using would have $p_{f, n}=0$ and hence zero SINR in those channels.

Similarly, the uplink SINR for link $m$ on subchannel $n$ is given by

$$
\gamma_{m, n}=\frac{p_{m, n} h_{m m, n}}{I_{m, n}+\sigma^{2}}
$$

where the uplink co-channel interference for MBS $m$ on subchannel $n$ is

$$
I_{m, n}=\sum_{f} p_{f, n} h_{f m, n}+\sum_{m^{\prime} \neq m} p_{m^{\prime}, n} h_{m^{\prime} m, n} .
$$

From Shannon theory, the capacity for femto link $f$ on subchannel $n$ is

$$
\mathcal{C}_{f, n}=\frac{B}{N} \log _{2}\left(1+\gamma_{f, n}\right)
$$

Similarly, the capacity for macro link $m$ on subchannel $n$ is

$$
\mathcal{C}_{m, n}=\frac{B}{N} \log _{2}\left(1+\gamma_{m, n}\right)
$$

Summing the capacity over all femto and macro links and over all subchannels, the total throughput of the macro-femto network is given by

$$
\mathcal{C}=\sum_{n=1}^{N}\left\{\sum_{f=1}^{F} \mathcal{C}_{f, n}+\sum_{m=1}^{M} \mathcal{C}_{m, n}\right\}
$$

Problem P1 (Power and Subchannel Allocation):

Given the positions of MUEs, MBSs, FUEs, and FAPs, and a power and subchannel allocation of MUEs allocation $p_{m, n}$ for all $m \in M$ and $n \in N$, determine an optimal power and subchannel allocation $p_{f, n}$ for all $f \in F$ and $n \in N$ that maximizes the total capacity subject to power constraint of each FUE, i.e.,

$$
\begin{gathered}
\max \mathcal{C} \\
\text { subject to } \sum_{n \in N} p_{f, n}=P_{T}, \quad \forall f \in F
\end{gathered}
$$

To simplify finding the solution to Problem P1, we assume that only one subchannel is selected per FUE. Hence, the optimal subchannel allocation for FUE $f$ is $p_{f, n}=P_{T}$ for only one channel $n \in N$ and all the other channels $n^{\prime} \neq n$ have $p_{f, n^{\prime}}=0$. In another word, we only consider the subchannel allocation problem $\mathrm{P} 2$, not the power allocation problem $\mathrm{P} 1$. The problem of joint power and subchannel allocation is difficult and normal approaches are to do a separate (and iterative) power allocation and subchannel allocation.

Problem P2 (Subchannel Selection): Given the positions of MUEs, MBSs, FUEs, and FAPs, and a power and subchannel allocation of MUEs allocation, determine an optimal subchannel allocation that maximizes the total uplink capacity $C$ of the femto and macro networks.

However, finding an optimal centralized solution to Problem P2 is not practical in networks with many femto and macro cells due to a large amount of information such as locations of the cells and messaging exchanges (see [14]). Hence, we are looking for a distributed or decentralized solution for Problem P2. The solution is distributed in the sense that each FUE $f$ is assumed to be able to sense and know the SINR of all subchannels $\gamma_{f, n}$ for all $n$. Each cognitive FUE does not know the locations of other FUEs, FAPs, MUEs, nor MBSs. Neither it knows about the selected subchannels of other femto or macro links.

\section{Best-Response Distributed Subchannel SElection Algorithm}

In this paper, we propose a distributed subchannel selection algorithm used by each FUE to attempt to solve the subchannel selection problem in Problem P2. To reduce overheads and allow for non-cooperative behaviors, we assume that FUEs do not exchange information about channel selection. However, each FUE can sense and adapt to its environmental changes by selecting a proper subchannel allocation. Provided with such sensing capabilities, each FAP can make its own decision of transmission on subchannel that will give them a better SINR.

Our distributed algorithm shown in Algorithm 1 is based on a game approach, following the concept of the best response strategy (see, e.g., [15], [5], [16]), where each player selects the best action that maximizes its own utility given the current strategies of all other players.

Specifically, we assume that all FUEs are synchronized and the time is divided into time-slots of a fixed duration. Each time-slot is further divided into two time durations or phases, called sensing phase and payload phase. During the sensing 
$\overline{\text { Algorithm } 1 \text { Best-Response Distributed Subchannel Selection }}$ Algorithm, run by each FUE

1) Initialization:

a) Get synchronization.

b) Select a subchannel $n_{0}$ randomly from the set $N$. Transmit during the sensing phase in subchannel $n_{0}$.

2) In sensing phase of time-slot $t=1,2, \ldots$ :

a) Measure $\operatorname{SINR}_{n}$ of every subchannel $n \in N$.

b) Select a subchannel $n_{t}^{\prime}$ with the highest SINR among $\left\{\operatorname{SINR}_{1}, \ldots, \operatorname{SINR}_{N}\right\}$.

3) In payload phase of time-slot $t$ :

a) Transmit data in subchannel $n_{t}^{\prime}$.

b) Set $t \leftarrow t+1$. Go to Step 2).

phase of time-slot $t$, each FUE senses the subchannels for their SINRs. During this sensing phase, all FUEs keep transmitting at their selected channels used in the previous time-slot $t-$ 1. The duration of the sensing phase should be minimized while providing a sufficiently accurate SINR measurement of all channels.

After getting the SINR measurement of all the subchannels, at the end of the sensing phase each FUE selects the subchannel with the highest SINR for transmission in the payload phase (the rest of time-slot $t$ ). By selecting the highest SINR subchannel, each FUE attempts to maximize its own channel capacity given the strategies or actions of other FUEs in the previous time-slot and assuming that other users will not change their selections at the next time-slot. This is a version of the best-response strategy.

Best-Response Strategy: The best-response strategy $b r_{i}\left(s_{-i}\right)$ of player $i$ to the profile of strategies $s_{-i}$ played by all other opponents, where $s_{-i}=$ $\left\{s_{1}, \ldots, s_{i-1}, s_{i+1}, \ldots, s_{N}\right\}$, is defined as [15]:

$$
b r_{i}\left(s_{-i}\right)=\arg \max _{s_{i} \in \mathcal{S}_{i}} u_{i}\left(s_{i}, s_{-i}\right)
$$

where $u_{i}$ is the utility of player $i$ anbd $\mathcal{S}_{i}$ is the set of possible strategies of player $i$.

In the next time-slot $t+1$, each FUE senses the SINR of all channels again during the sensing phase and chooses the channel with the highest SINR for transmission in the payload phase for that time-slot $t+1$. This process is repeated forever and we have a repeated best-response strategy [5]. For example, if an FUE $f$ uses a subchannel $n_{f, t}$ during the payload phase of time-slot $t$, it will still transmit at subchannel $n_{f, t}$ during the sensing phase of time-slot $t+1$. However, FUE $f$ may use another subchannel $n_{f, t+1}$ during the payload phase of time-slot $t+1$.

Since the channel used in the payload phase of the current time-slot is determined from the SINRs, resulted from the channels in the previous time-slot and the FUEs update their channel selection simultaneously (hence, they are making a simultaneous move), there could be oscillations between some sets of strategies.

\section{A. An Example of Oscillating Behavior}

Let us analyze a simple example to gain more understanding of the problem. Consider an example with only two subchannels, no macro links, and three femto links. For simplicity, we assume all FUEs are co-located, all FAPs are co-located, and the same Gaussian noise levels. Hence, all femto links are homogeneous, i.e., they have the same received power and the same signal-to-noise ratio (SNR). We call this example a 3-link, 2-ch, homogeneous case. For this case there are 8 possible channel selections: the two same-channel selections for the three users are 111 and 222 and the six mixed-channel selections are $112,121,211,122,212$, and 221 .

Regarding the instantaneous total capacity, each samechannel selection has the same instantaneous total capacity, ideally given by the Shannon's capacity

$$
C_{\text {same }}=3 \log _{2}\left(1+\frac{P}{2 P+N}\right)
$$

bps/Hz, where $P$ denotes the received signal power and $N$ the received noise power. In this same-channel selection, each user receives the same individual capacity. For the mixed-channel selections, each selection also has the same instantaneous total capacity of

$$
C_{\text {mix }}=\log _{2}\left(1+\frac{P}{N}\right)+2 \log _{2}\left(1+\frac{P}{P+N}\right)
$$

Here the user with the channel different from the others has higher capacity of $\log _{2}\left(1+\frac{P}{N}\right)$, while the other two users each has capacity $\log _{2}\left(1+\frac{P}{P+N}\right)$.

Let's now consider two different scenarios that result in oscillation of channel selections:

1) Scenario 1: If initially at time $t=0$, all FUEs start with channel 1 , then during the sensing phase of time $t=1$, they would all see that the SINR of channel $1\left(\operatorname{SINR}_{1}=\frac{P}{2 P+N}\right)$ is less than that of channel $2\left(\operatorname{SINR}_{2}=\frac{P}{N}\right)$ and hence switch to channel 2 during the payload phase of time $t=1$. But in the sensing phase of $t=2$, they all would see that $\mathrm{SINR}_{1}=\frac{P}{N}>\mathrm{SINR}_{2}=\frac{P}{2 P+N}$ and hence switch to channel 1. Hence, there is an oscillation between all FUEs using channel 1 and all using channel 2. Although the channel selections are alternating between 111 and 222, the instantaneous total capacity in each time-slot is constant at $C_{\text {same }}$.

2) Scenario 2: If instead, the initial channel selection at $t=0$ is one of the six mixed-channels, say 112 , then at the sensing phase of $t=1$, all users sense that channel 2 has better SINR than channel 1 and then all use channel 2 during the payload phase of $t=1$, and then we fall into the oscillation of Scenario 1, i.e., the sequence of channel selections is $112 \rightarrow$ $222 \rightarrow 111 \rightarrow 222 \rightarrow 111 \rightarrow \ldots$

Hence, no matter of what initial channel selections, oscillations always occur if each user simultaneously follows the 
best-response in Algorithm 1, due to the simultaneous moves and the fact that each user ignores the possibility that the other users can also switch channels in the next time-slot.

\section{B. Mixed Nash Equilibrium and Price of Anarchy}

For the example of the 3-link, 2-ch, homogeneous case, the instantaneous total capacity of the mixed-channel initial selection is higher than that of the same-channel initial selection only in the initial time-slot, but the average total capacity over a large number of time-slots in both scenarios is equal to $C_{\text {mix }}$. For this homogeneous example, although there is no static Nash Equilibrium (NE), there is a mixed NE (see [17]), where each user randomly but equally chooses between the two subchannels, independent of the sensed SINR. This results in a Markov chain where over the long run, the mixed NE assigns a probability $1 / 8$ to each of the 8 sets of all possible channel selections. The instantaneous total capacity is oscillating between two values: $C_{\mathrm{mix}}$ and $C_{\text {same }}$ (where $C_{\text {same }}$ happens 0.25 of the time), giving the average total capacity of the mixed $\mathrm{NE}$ as

$$
C_{\mathrm{NE}}=0.25 C_{\text {same }}+0.75 C_{\text {mix }}
$$

This capacity is between the worst capacity $C_{\text {same }}$ and the best capacity $C_{\mathrm{mix}}$, which could occur when the users are assigned the channels by a centralized scheduler.

Fig. $2 \mathrm{a}$ plots $C_{\text {same }}, C_{\text {mix }}$, and $C_{\mathrm{NE}}$ for varying signal-tonoise ratio $P / N=\mathrm{SNR}$. It is obvious that the capacity $C_{\mathrm{NE}}$ of the mixed NE strategies is lower than $C_{\text {mix }}$ of the centralized strategy. The lower performance of the mixed NE strategies is due to the selfishness of each FUE or the bestresponse strategy. In game theory, one way to measure the efficiency of Nash equilibrium is via the (mixed) price of anarchy (PoA), which is the ratio between the performance of the worst mixed Nash equilibrium to that of the optimal centralized strategy [18]. In this example, there is only one mixed Nash equilibrium and the PoA is

$$
\text { PoA }=\frac{C_{\mathrm{NE}}}{C_{\mathrm{mix}}}=0.25 \frac{C_{\mathrm{same}}}{C_{\mathrm{mix}}}+0.75
$$

as shown in Fig. 2b Note that as $S N R=P / N \rightarrow \infty$, we have $C_{\text {mix }} \rightarrow \infty$ and $P o A \rightarrow 0.75$ since $C_{\text {same }}$ becomes a constant at high SNR (i.e., $C_{\text {same }} \rightarrow 3 \log _{2}(1+P / 2 P)=1.755$ bps/Hz). Hence, the minimum PoA is 0.75 .

\section{Avoiding Oscillation using Stochastic Best-Response}

One way to avoid the oscillation problem is avoiding the simultaneous move. This could be done by a sequential move, where each user updates its channel selection one at a time, in a round-robin fashion, i.e., the sequence of updates is user 1 , user 2, user 3, then user 1 again. Hence, the same-channel selection is always avoided and the average total capacity is $C_{\text {mix }}$ which is the maximum. However, this requires some coordination among the users

To keep our proposed algorithm distributed, we instead avoid the simultaneous moves by having each user updates

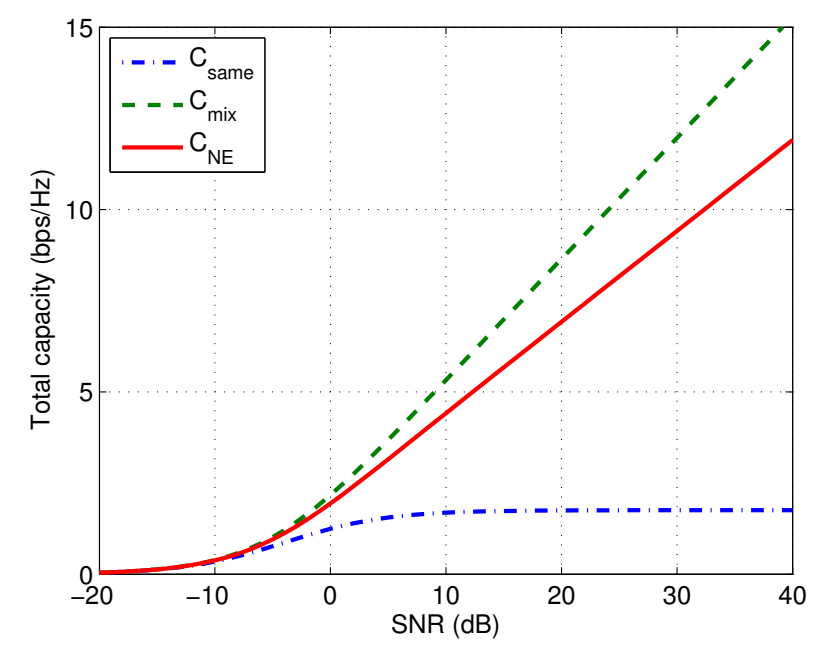

(a) Total capacity

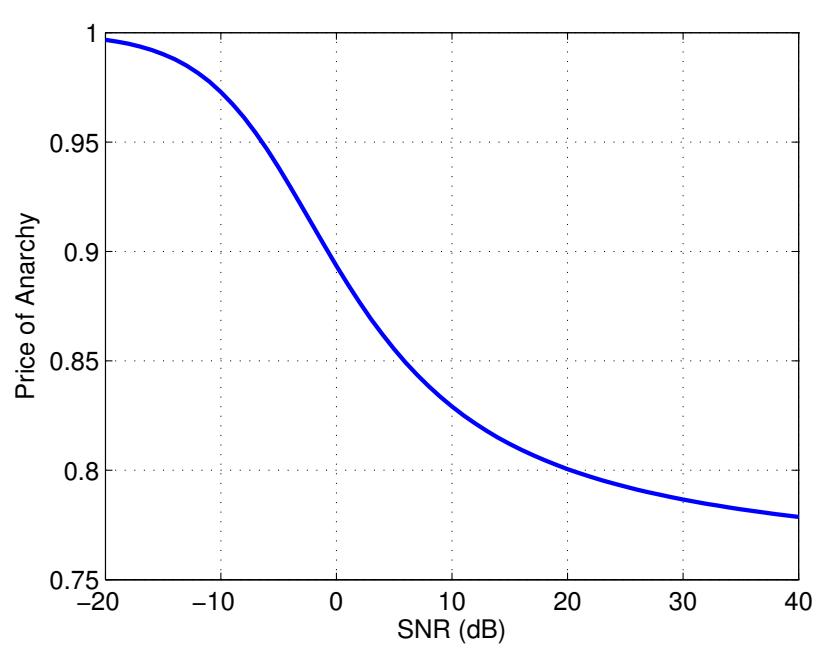

(b) Price of Anarchy

Fig. 2: The example of three homogeneous femto links and two channels: (a) The instantaneous total capacity $C_{\text {same }}$ and $C_{\text {mix }}$ and the average total capacity for the mixed NE strategies $C_{\mathrm{NE}}$ for varying signal-to-noise ratio $\mathrm{SNR}=P / N$. (b) the Price of Anarchy.

its channel selection decision independently, without coordination, with a probability $p$. Hence, Algorithm 1 is revised to be Algorithm 2, with the addition of Step 2.c. In Step 2.c, the new channel with the highest SINR is selected with probability $p$, otherwise the previous channel is used again. We call this revised best-response a stochastic best-response.

This stochastic best-response is a version of a best-response play but with uncertainty of updates or outcomes (e.g., see [19], [20], [21]). Here, we assume that each player plays the best-response strategy with probability $p$ if the current strategy is not a best-response one. However, if it is already a bestresponse, stay with it. Hence, the deterministic best-response strategy is played stochastically. 
$\overline{\text { Algorithm } 2 \text { Stochastic Best-Response Distributed Subchan- }}$ nel Selection Algorithm, run by each FUE

1) Initialization:

a) Get synchronization.

b) Select a subchannel $n_{0}$ randomly from the set $N$. Transmit during the sensing phase in subchannel $n_{0}$.

2) In sensing phase of time-slot $t=1,2, \ldots$ :

a) Measure $\operatorname{SINR}_{n}$ of every subchannel $n \in N$.

b) Select a subchannel $n_{t}^{\prime}$ with the highest SINR among $\left\{\operatorname{SINR}_{1}, \ldots, \operatorname{SINR}_{N}\right\}$.

c) Draw a uniform $[0,1]$ random variable $u$. If $u \leq p$, set $n_{t}=n_{t}^{\prime}$; otherwise, $n_{t}=n_{t-1}$.

3) In payload phase of time-slot $t$ :

a) Transmit data in subchannel $n_{t}$.

b) Set $t \leftarrow t+1$. Go to Step 2).

1) Modeling Stochastic Best Response as a Markov Chain: The behavior of the stochastic best-response can be done using a Markov chain, where the states are the eight different channel selections in $\mathcal{S}=\{111,112, \ldots, 222\}$. It is a Markov chain because the future state at time-slot $t+1$ depends only on the current state at time-slot $t$. With the probability $p$, all transition probabilities can be determined.

Example 1: If the current state is 111, then using deterministic best response the new state should be 222 , i.e., every user wants to switch to channel 2. However, recall that each can make a switch with only probability $p$. Suppose all users can make the switch, i.e., each can draw $u \leq p$ in Step 2.c in Algorithm 2. This happens with probability $p^{3}$. Hence, the transition probability from state 111 to state 222 is

$$
P_{(11) \rightarrow(222)}=p^{3} \text {. }
$$

However, if only users 1 and 3 get draws more than $p$ in Step 2.c while the user 2 cannot, the new state is 212 and happens with probability $p \times q \times p$ where $q=1-p$ is the probability of not success in switching. Hence,

$$
P_{(111) \rightarrow(212)}=p^{2} q .
$$

Example 2: If the current state is 212, then from the deterministic best-response the new state should be 111, i.e., only users 1 and 3 want to switch to channel 1 . However, suppose only user 1 succeeds in switching, the new state is instead 112 and happens with probability $p \times 1 \times q$, where the probability for user 2 to use channel 1 is 1 since it always uses channel 1 independent of the outcome of the draw in Step 2.c. Hence, we have

$$
P_{(212) \rightarrow(112)}=p q .
$$

A similar reasoning gives $P_{(212) \rightarrow(111)}=p^{2}$. Note that since from the best-response of user 2 , it stays with channel 1 , the probability of its moving to channel 2 in the next state is 0 , i.e.,

$$
P_{(212) \rightarrow(i 2 j)}=0
$$

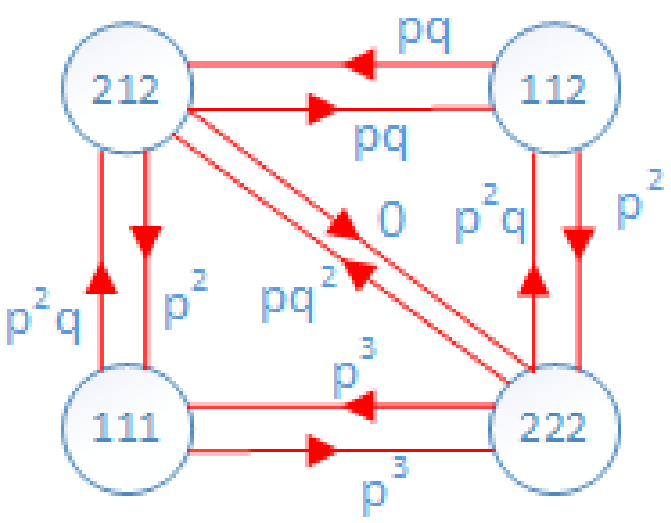

Fig. 3: Illustration of a part of the Markov chain

for any $i, j=1,2$.

Other transition probabilities can be calculated similarly. A part of the resulted Markov chain is shown in Fig. 3 for illustration.

2) Analysis of the average total capacity: For $0<p<1$, the Markov chain is recurrent and aperiodic and hence ergodic. Hence, in the long run the stochastic best-response reaches a steady strategy distribution, independent of the initial state. The steady-state probability distribution $\pi=$ $\left[\pi_{1}, \pi_{2}, \ldots, \pi_{8}\right]$ where states $111,112, \ldots, 222$ are now called states $1,2, \ldots, 8$, respectively, must satisfy $\pi=\pi Q$ and $\sum_{i=1}^{8} \pi_{i}=1$ where $Q=\left[P_{i \rightarrow j}\right]$ is the transition probability matrix and $P_{i \rightarrow j}$ is the transition probability from state $i$ to state $j$ for $i, j=1,2, \ldots, 8$.

Since the Markov chain is ergodic, the average total capacity over the long run for the switching probability $p$ is equal to the average capacity calculated with the steady-state probabilities $\pi$, as

$$
C_{p}=\sum_{i=1}^{S} C_{i} \pi_{i}
$$

where $C_{i}=C_{\text {same }}$ for $i=1,8$ and $C_{i}=C_{\text {mix }}$ otherwise. The steady-state probability vector $\pi$ is a function of $p$.

\section{NumericAl RESUlts}

In this section we evaluate by simulation the performance of the proposed algorithm for different selection probability $p$, and density. For simulation, we assume the channel model is deterministic and uniform across channels, with the channel gain between FUE $f^{\prime}$ and FAP $f$ in any subchannel $n$ is

$$
h_{f^{\prime} f, n}=\min \left\{d_{f^{\prime} f}^{-\alpha}, h_{\max }\right\}
$$

where $d_{f^{\prime} f}$ is the distance between $f^{\prime}$ and $f, h_{\text {max }}$ is the allowable maximum gain, and the constant $\alpha \in[2,4]$.

\section{A. Homogeneous 3-Link, 2-Channel Case}

We simulate a homogeneous 3-femto-link, 2 channel case as described in Section IV-A to confirm our analysis and the steady-state distribution and the average total capacity. 


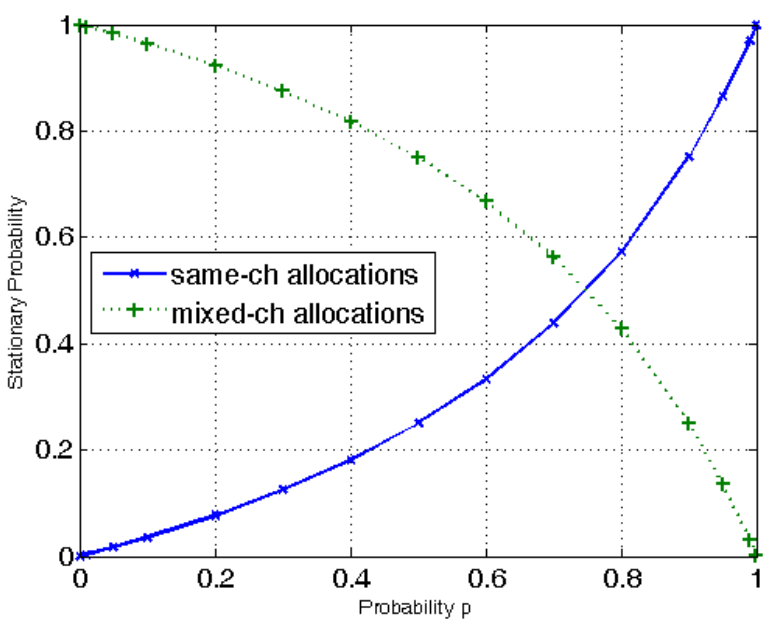

Fig. 4: Plot of stationary probability distribution of the samechannel states (states 111 and 222) and the mixed-channel states vs probability $p$.

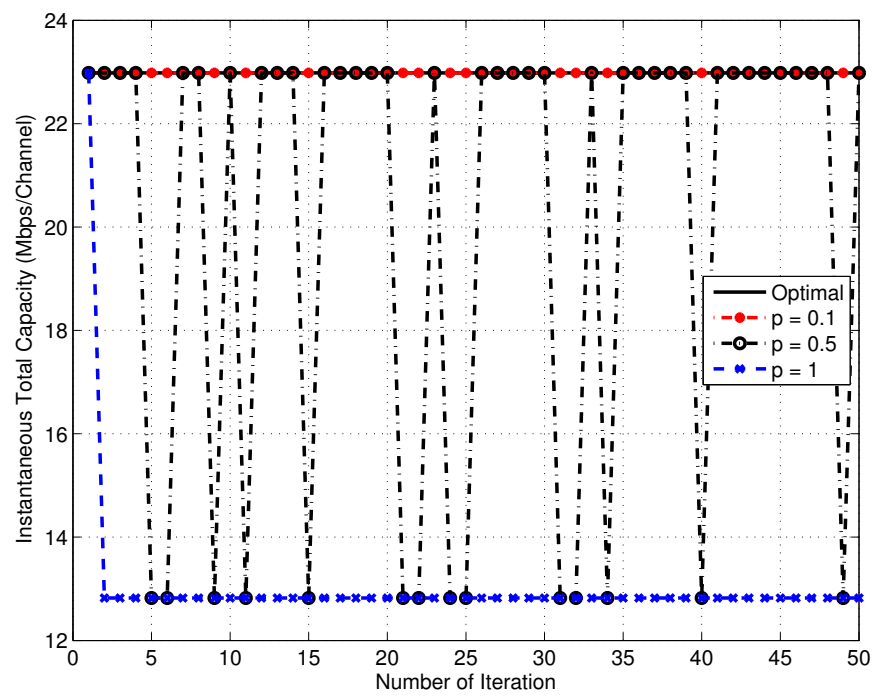

Fig. 5: Instantaneous total capacity (Mbps/Channel) of macrofemto networks with 3 femto links and 2 subchannels.

Each of the three FUEs are at $30 \mathrm{~m}$ distance from its FAP. The transmit power is 1 watt, the receive noise power is 1 milliwatt, and each subchannel is $10 \mathrm{MHz}$. This gives the receive power $P=1000 / 30^{2} \mathrm{~mW}$ and $\mathrm{SNR}=P / N=0.458$ $\mathrm{dB}$. From calculation, we have $C_{\text {same }}=12.82, C_{\text {mix }}=22.98$ and $C_{\mathrm{NE}}=20.44 \mathrm{Mbps}$, respectively.

From the analysis of the steady-state probability distribution of the Markov chain, the stationary probability distribution of the same-channel states (states 111 and 222) and the mixedchannel states vs probability $p$ are shown in Fig. 4 . Fig. 5 shows the instantaneous total capacity vs iterations (or timeslots) for $p=0.1,0.5$ and 1 . Fig. 6 shows the average total capacity over a long run of the simulation and that from calculation from the Markov chain.

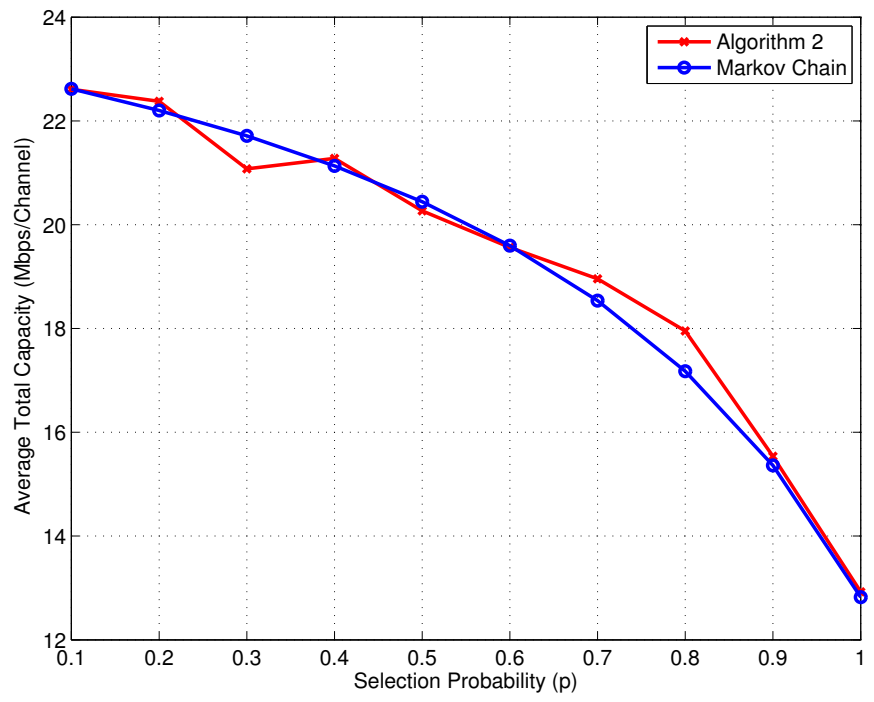

Fig. 6: Comparison of average total capacity vs. selection probability $p$ (varies from 0.1 to 1 ), using Algorithm 1 and Markov chain.

For $p=1$, each user plays the deterministic best-response and hence there is an oscillation in the channel selections between 111 and 222. The instantaneous total capacity is always at $C_{\text {same }}$ and hence the average total capacity $C_{p}=C_{\text {same }}$ as shown in Fig. 6

For $p=0.5$, Fig. 4 shows that the channel selections are $75 \%$ of the time in the mixed-channel state. This is the same distribution of the strategies as the mixed NE, although the stochastic best-response is different from the mixed NE. Hence, the average total capacity for $p=0.5$ is the same as that of the mixed NE, i.e.,

$$
C_{(p=0.5)}=C_{\mathrm{NE}}
$$

Here the probability that exactly one user updates its channel are $3 * 0.5^{3}=0.375$ and that of exactly two users updates their channels also 0.375 .

For $p=0.1$, each user rarely switches to the new channel. The probability that exactly one, two and three users update their channels is $3 * 0.1 * 0.9^{2}=0.243,0.027$, and 0.001 , respectively. Hence, in most of the time-slots, we have at most one user switches its channel selection. This stochastic bestresponse with a small value of $p$ gives a similar affect as a round-robin sequential updates used to avoid simultaneous move problem (discussed early in Section IV-C). However, the stochastic best response does not require any explicit coordination, although this comes with a price of a longer time to achieve the steady state distribution.

\section{B. Homogeneous 5-Link, 3-Channel Case}

Here we generalize the homogeneous 3-link, 2-channel case a little to 5 links and 3 channels, where now we have 5 links and all FUEs are co-located and all FAPs are also co-located. From calculation of the instantaneous total capacities, there are 5 different capacity values. That is, the lowest total capacity 


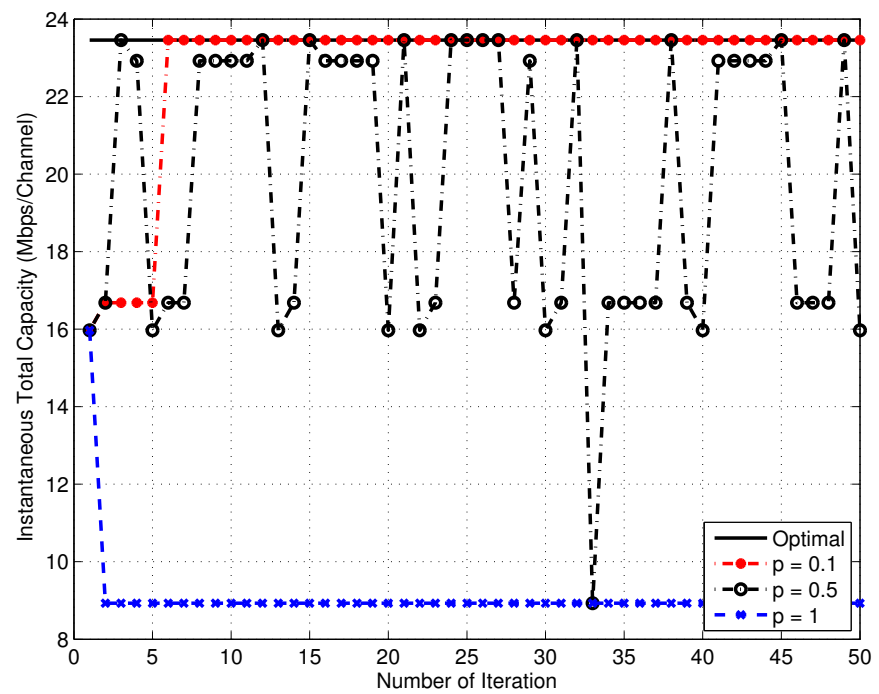

Fig. 7: Instantaneous total capacity (Mbps/Channel) of macrofemto networks for 5 femto links and 3 subchannels.

happens when all 5 users in the same channel (i.e., for the channel selections 11111, 22222, and 33333) and the total capacity is $5 \log _{2}\left(1+\frac{P}{4 P+N}\right)$. The next higher total capacity is when 4 users in one channel and the other in another channel (e.g., for the channel selection 11112) and the total capacity is $4 \log _{2}\left(1+\frac{P}{3 P+N}\right)+\log _{2}\left(1+\frac{P}{N}\right)$. We can follow this way of thinking and get 5 different total capacity values, where the highest one happens when 2 users in one channel, other 2 users in another channel and the last user in yet another channel (e.g., the channel selection 11223). This gives the highest total capacity of $4 \log _{2}\left(1+\frac{P}{P+N}\right)+\log _{2}\left(1+\frac{P}{N}\right)$.

Fig. 7 shows the instantaneous total capacity vs iterations for $p=1,0.5$ and 0.1 . As expected there are 5 different capacity values. With the values of $P$ and $N$ as in the 3-link, 2-channel case and the channel bandwidth of $20 / 3 \mathrm{MHz}$, the five total capacities are calculated as $8.93,15.97,16.68,22.92$, and 23.45 Mbps, respectively.

At $p=1$, the stochastic best-response becomes deterministic best-response and an oscillation occurs. Just like in the 3 link 2 channel case, we can deduce that any initial condition would result in oscillation. An example of the sequence of channel selections is $11223 \rightarrow 33333 \rightarrow 11111 \rightarrow 22222 \rightarrow$ $33333 \rightarrow \ldots$ Here we assume that when more than one channels have the highest SINR, the user selects the one with the lowest index. However, if the user randomly chooses one out of the best channels, then the sequence would be something like $11223 \rightarrow 33333 \rightarrow 12211 \rightarrow 33333 \rightarrow$ $22211 \rightarrow 33333 \rightarrow \ldots$, which result in the same channel allocation 33333, half of the times. The detailed study for a more number of homogeneous links and channels is a future work.

\section{Effects of More Number of Links}

Here we fix the number of channels to five and vary the number of homogeneous links $N$ to be 10, 20 and 30. The

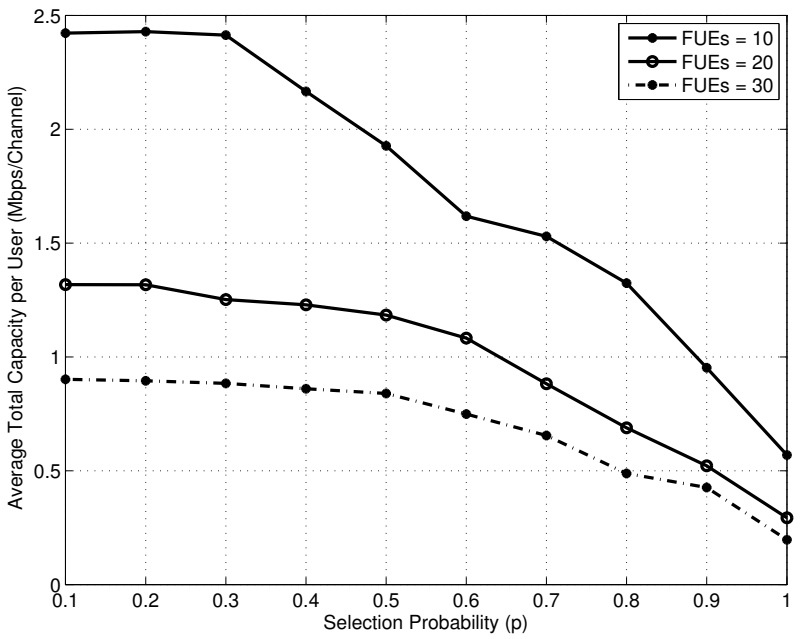

Fig. 8: Comparison of average total capacity per user vs. selection probability $p$ for varying number of FUEs.

FUEs are co-located and same as FAPs. The average total capacities per user for different $N$ are shown in Fig. 8 for different selection probability $p$. The average total capacity per user decreases with the number of links $N$ and it consistently decreases with an increase in the selection probability $p$, as we have observed for the 3-link, 2-channel and 5-link, 3-channel cases.

\section{CONCLUSION}

In this paper, we proposed a distributed algorithm for subchannel selection by non-cooperative femtocell users in macro-femto networks. With restriction on information exchange among other femtocells, each femtocells relies on SINR at various subchannels and then responds by selecting the subchannel corresponding to highest-SINR following our algorithm. Each user regularly senses the SINRs on all subchannels and selects the best subchannel. We discuss in details the case of homogeneous 3-link, 2-channel case. If each user selects the best channel following a deterministic best-response, an oscillation occurs no matter of the initial subchannel selection. This oscillation gives the lowest total capacity. To avoid the oscillation and achieve the highest total capacity, while still assuming no coordination among the users, we propose a stochastic best-response algorithm and use a Markov chain to analyze its average capacity performance.

Using simulation results, we confirm our analysis of the Markov chain and also provide performance of other homogeneous cases with more number of homogeneous links and subchannels. It is shown that the highest total capacity can be achieved when the selection probability $p$ is small. This stochastic best-response with small $p$ in effect provides a sequential move to update each decision and hence avoids the problem of simultaneous moves.

Some future works could include a more detailed study of the performance of the stochastic best-response for homogeneous links but with any number of links and subchannels and 
more general cases of non-homogeneous links. Furthermore, to avoid the oscillation of the subchannel selections, other methods such as having each user expect the possibility that other users can also update their subchannel selections should also be considered.

\section{REFERENCES}

[1] FP7-ICT-2009-4, "Femtocell-based network enhancement by interference management and coordination of information for seamless connectivity." [Online]. Available: http://www.ict-freedom.eu/

[2] V. Chandrasekhar, J. Andrews, and A. Gatherer, "Femtocell networks: a survey," IEEE Commun. Mag., vol. 46, no. 9, pp. 59-67, 2008.

[3] Small-Cell, "Small cell forum unlocks enterprise opportunity for mobile operators." [Online]. Available: http://www.smallcellforum.org/

[4] L. T. W. Ho and H. Claussen, "Effects of user-deployed, co-channel femtocells on the call drop probability in a residential scenario," in IEEE 18th Int. Symp. on Personal, Indoor and Mobile Radio Commun. (PIMRC 2007), 2007, pp. 1-5

[5] R. Engelberg, A. Fabrikant, M. Schapira, and D. Wajc, "Best-response dynamics out of sync: complexity and characterization," in Proc. of the 14th ACM conf. on Electron. commerce. ACM, 2013, pp. 379-396.

[6] A. D. Jaggard, M. Schapira, and R. N. Wright, "Distributed Computing with Adaptive Heuristics," ArXiv e-prints, Oct. 2009.

[7] D. Niyato and E. Hossain, "Competitive spectrum sharing in cognitive radio networks: a dynamic game approach," IEEE Trans. on Wireless Commun., vol. 7, no. 7, pp. 2651-2660, 2008.

[8] C. Seneviratne and H. Leung, "A game theoretic approach for resource allocation in cognitive wireless sensor networks," in IEEE Int. Conf. on Syst., Man, and Cybern., 2011, pp. 1992-1997.

[9] J. Dong, D. B. Smith, and L. W. Hanlen, "Co-channel interference mitigation for wireless body area networks coexistence using a non-cooperative game," CoRR, vol. abs/1408.0570, 2014. [Online]. Available: http://arxiv.org/abs/1408.0570
[10] I. Mustika, K. Yamamoto, H. Murata, and S. Yoshida, "Potential game approach for self-organized interference management in closed access femtocell networks," in IEEE 73rd Veh. Tech. Conf., 2011, pp. 1-5.

[11] M. Nazir, M. Bennis, K. Ghaboosi, A. MacKenzie, and M. Latvaaho, "Learning based mechanisms for interference mitigation in selforganized femtocell networks," in 44th Asilomar Conf. on Signals, Syst. and Comput. (ASILOMAR), 2010, pp. 1886-1890.

[12] E. J. Hong, S. Y. Yun, and D.-H. Cho, "Decentralized power control scheme in femtocell networks: A game theoretic approach," in IEEE 20th Int. Symp. on Personal, Indoor and Mobile Radio Commun., 2009, pp. 415-419.

[13] C. Shi, R. A. Berry, and M. L. Honig, "Distributed interference pricing for ofdm wireless networks with non-separable utilities," in 42nd Annu. Conf. on Inform. Sci. and Syst., 2008, pp. 755-760.

[14] M. Dinitz, "Distributed algorithms for approximating wireless network capacity," in Proc. of the 29th Conf. on Inform. Commun., ser. INFOCOM'10. Piscataway, NJ, USA: IEEE Press, 2010, pp. 1397-1405.

[15] M. Felegyhazi and J.-P. Hubaux, "Game theory in wireless networks: A tutorial," Tech. Rep., 2006.

[16] N. Nisan, M. Schapira, G. Valiant, and A. Zohar, "Best-response mechanisms," in ICS, 2011, pp. 155-165. [Online]. Available: http://www.cs.huji.ac.il/ noam/BRM.pdf

[17] D. Fudenberg and J. Tirole, Game Theory. MIT Press, 1991.

[18] E. Koutsoupias and C. Papadimitriou, "Worst-case equilibria," Comput. sci. review, vol. 3, no. 2, pp. 65-69, 2009.

[19] R. D. McKelvey and T. R. Palfrey, "Quantal response equilibria for normal form games," Games and Economic Behavior, vol. 10, no. 1, pp. 6-38, 1995.

[20] T. Ui, "Quantal response equilibria and stochastic best response dynamics," Yokohama National University, Tech. Rep., 2002.

[21] D. Oyama, W. H. Sandholm, and O. Tercieux, "Sampling best response dynamics and deterministic equilibrium selection," Theoretical Econ., 2014. [Online]. Available: http://EconPapers.repec.org/RePEc: the:publsh:1405 